\title{
Quantitative Analysis of Crude Plasma Extracts by Ion Trap Tandem Mass Spectrometry
}

\author{
Robert J. Strife and John R. Simms \\ Miami Valley Laboratories, The Procter and Gamble Co., Cincinnati, Ohio, USA
}

The ion trap mass spectrometer is a tandem-in-time instrument that has promise as an extremely sensitive device for practical tandem mass spectrometry assays. An approach for the quantitative analysis of unknown drug levels in crude extracts, using combined capillary gas chromatography and the ion trap mass spectrometer in the tandem mode, is described. One-gram plasma samples were spiked with an anti-inflammatory drug at levels of 1-100 $\mathrm{ng}$, and with $50 \mathrm{ng}$ of a chemical analog internal standard. Crude extracts of the plasma samples are analyzed by using scan functions that utilize combined radiofrequency (rf) and dc voltages. The need for combined rf- and dc-voltage sequences for analysis of such extracts is demonstrated by comparison to attempted analyses using only if voltages. Limitations of the method are: (1) the need for accurate calibration of ionization times to obtain linear calibration lines, and (2) the lack of automatic gain control for scans using combined $\mathrm{rf}$ and $\mathrm{dc}$ voltages to control and optimize parent ion populations and to allow a simpler analysis of "unknowns." (J Am Soc Mass Spectrom 1992, 3, 372-377)

$\mathrm{T}$ he ion trap mass spectrometer (Finnigan-MAT, San Jose, CA) is known to have high "observed sensitivity" in the combined capillary gas chromatography/mass spectrometry (GC/MS) mode, particularly when mass-selective storage is used. When switching to the combined capillary GC/tandem mass spectrometry (MS/MS) mode (referred to within as GC/MS/MS and restricted to the full product ion scan of a mass-selectively stored parent ion), sensitivity is retained. This is because the parent ions are converted with over $50 \%$ observed collision-induced dissociation (CID) efficiency in many cases [1].

What is less clear is if traps will have practical advantages over triple quadrupole devices for quantitative GC/MS/MS assays. This is especially true in regard to observed sensitivity for a target analyte [1], if single reaction monitoring is sufficient for the analysis. Therefore, a study using the iun trap as a quantitative GC/MS/MS tool has been undertaken, with a goal of comparing results to those from a triple quadrupole tandem mass spectrometer.

The steps of a MS/MS experiment are carried out stepwise, in time, by using the ion trap mass spectrometer. In our case these can be described as: (1) creation and trapping of a population of ions with a range of mass-to-charge ratio values; (2) subsequent, more selective storage of a $2-3 \mathrm{u}$ range, including the

Address reprint requests to Robert J. Strife, Miami-Valley Laboratories, The Procter and Gamble Co., P.O. Box 398707, Cincinnati, OH $45239-8707$. parent ion; (3) resonant excitation of only the parent ion to cause CID; and (4) ejection and detection of the formed product ions. The total time for such an experiment is typically $50 \mathrm{~ms}$, well within the elution time frame of compounds undergoing capillary GC. The observed CID efficiencies are on the order of 50-100\% for positive parent ions produced by electron ionization (EI) $[2,3]$.

The quantitation of analytes at the parts-per-billion level in crude extracts has certain problems unique to ion traps. Most significant, the trap has a limited capacity to store ions. If too many ions are created prior to a scan, space charge effects can be deleterious to subsequent steps of analysis. However, in trace analysis of a crude extract, the analyte is by definition a minor component in a large amount of matrix. Using EI pulses to produce adequate signals for program-nanogram of analyte standards inevitably leads to space charge when the analyte is in a crude extract.

The possibility of ion-molecule reactions between the desired parent ion and neutral matrix molecules, prior to MS/MS, must also be considered. These thermodynamically controlled reactions could cause depletion of parent ion populations $[4,5]$. This problem can be evaluated by comparing signals oblained from standards with those from the same material spiked into crude extracts. In our work reported so far we do not believe this has been a problem.

We previously explained the necessity of using scan functions with combined radiofrequency (rf) and pulsed dc voltages (referred to within simply as $\mathrm{rf} / \mathrm{dc}$ ) for qualitative analysis of crude tissue extracts [6]. In 
the $\mathrm{rf} / \mathrm{dc}$ mode, ions of a given 2-3 $\mathrm{u}$ mass range are stored, while all others are ejected [3, 7-9]. Initial space charge is reduced while analyte ions are efficiently stored. However, more complex methods like rf/dc do not have any type of automatic gain control (AGC). The AGC mode, among other functions, allows the determination of concentrations of an analyte in "unknowns" [10, 11]. Moreover, the fuxed ionization times that can be chosen for $\mathrm{rf} / \mathrm{dc}$ work, via the software, must be known accurately to obtain calibration lines with high linearity. These limitations and initial attempts to overcome them are discussed within, in relation to practical GC/MS/MS analysis of spiked and clinical plasma samples.

\section{Experimental}

\section{Materials and Method}

Tebufelone (TE), formally 1-[3,5-bis $(1,1-$ dimethylethyl)-4-hydroxyphenyl]-5-hexyn-1-one, and a 3-methyl hexynone analog were synthesized, purifed, and fully characterized at Procter and Gamble's Miami Valley Laboratories. Various scan functions and procedures to establish calibration lines for the ion trap mass spectrometer, using pure standards and a 100:1 split ratio during sample introduction, have been previously described [12]. That is, only $1 \%$ of a 1-2 $\mu \mathrm{L}$ injection entered the capillary column. In this work GC/MS/MS determinations of "unknowns" were done, using the 3-methyl analog as the internal standard, with total introduction of a $2-\mu \mathrm{L}$ volume via splitless injection.

Two sets of calibration samples were prepared. The first set was derived from pure standards by adding $10 \mu \mathrm{L}$ of a solution containing from 1 to $100 \mathrm{ng}$ of TE and $10 \mu \mathrm{L}$ of a solution containing $54 \mathrm{ng}$ of internal standard directly to sample vials ( $n=5$, or five replicates). These samples were evaporated to dryness and were reconstituted in $20 \mu \mathrm{L}$ of acetone. This set of samples was used to check the linearity of calibration and to establish approximate response factors for the compounds. The second set of samples was derived from plasma.

Human plasma and clinical samples (HIV negative) were obtained within Miami Valley Laboratories and were handled with universal precautions. The extraction procedure was previously validated for a clinical assay based on triple quadrupole MS/MS, which has been fully discussed by Dobson et al. [13]. To obtain a calibration line from plasma, 1-g samples were spiked with $10 \mu \mathrm{L}$ aliquots of 'I'E and the internal standard, as described above $(n=3)$. Proteins were precipitated with acetonitrile and, following centrifugation, the supernatant was extracted with diethyl ether. The ether layer was evaporated and the samples were reconstituted in $20 \mu \mathrm{L}$ of acetone. Blanks were also processed similarly.
Samples of $2 \mu \mathrm{L}$ (10\% of a plasma extract) were injected splitless. This corresponds to $.080 \mathrm{ng}-8.0 \mathrm{ng}$ analyte and $4.3 \mathrm{ng}$ internal standard on-column, based on $80 \%$ recovery in the extraction process. Clinical samples previously analyzed by the TSQ method were submitted "blind" for analysis on the ion trap mass spectrometer. They were treated in a similar fashion, except that they were only spiked with the internal standard solution.

\section{Instrumentation}

The GC equipment and the ion trap mass spectrometer used in this work have been described [12]. EI pulse times indicated by the software were checked against oscillographic determinations of the gate potential as a function of time. The ion trap mass spectrometer scan function was similar to that described previously for GC/MS/MS work [2], Axial modulation $(6 \mathrm{~V})$ is routinely optimized and used during ejection and detection of daughter ions on our system $(v=530,000 \mathrm{~Hz}$, approximately [14]).

\section{Results and Discussion}

The clinical GC/MS/MS assay for TE had been previously developed, validated, and applied to thousands of analyses [13]. The purpose of the present work is to further the development of quantitative GC/MS/MS methodology on the ion trap mass spectrometer. The success of the ion trap mass spectrometer method is measured by comparison to an assay that has already been well defined on a triple quadrupole tandem mass spectrometer.

\section{Selection of Internal Standard for Trap Work}

Both stable isotope-labeled and analog internal standards were available for this project. Previously we showed several different approaches to obtaining calibration lines from an ion trap device [12]. Our conclusions were that superior results are obtained with internal standards separated by retention time. Similar observations have been subsequently made by others $[15,16]$. The details will not be reiterated here. Improvements allowing the use of stable isotope internal standards for quantitative GC/MS/MS on the ion trap mass spectrometer are still needed.

\section{Chromatography and Mass Spectra}

The chromatographic separation and mass spectra of TE and the 3-methyl hexynone analog (Figure 1) have been previously discussed $[12,13]$. The retention times are adjusted to typically fall between $5.5-6.5 \mathrm{~min}$ on a $15-\mathrm{m}$ column, with a separation of $.2 \mathrm{~min}$ between TE and the analog. In both of their mass spectra an intense radical cation of $m / z 248$ (100\% relative abundance) is produced by McLafferty rearrangement, cleaving the bond between the 2 and 3 positions of 

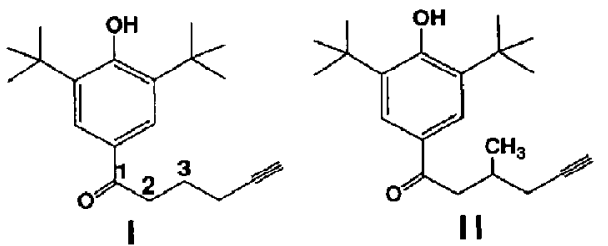

Figure 1. Structures of TE (I) and the 3-methyl analog (II) used as an unlabeled internal standard.

the side-chain. MS/MS of $m / z 248$ produces only $m / z 233$ with $100 \%$ observed CID efficiency for both compounds.

The observed instrumental sensitivity for this analyte is very good. Figure 2 shows a GC/MS trace (mass selective storage of $m / z 248$ using $\mathrm{rf} / \mathrm{dc}$ ) for $500 \mathrm{fg}$. The GC/MS/MS analysis $\left(248^{+} \rightarrow 233^{+}\right)$gives even better signal-to-noise ratio because the GC/MS analysis is limited by chemical background, albeit very low. Thus, we anticipated that the $80 \mathrm{pg}$ of analyte injected for the low concentration plasma calibration sample ( $1 \mathrm{ng} / \mathrm{g}$ ) would give adequate results.

\section{Selection of Ionization Times}

In GC/MS analyses on the ion trap, the ionization pulse time to be used for each recorded scan is tested by the data system in a short prescan. The produced ion current is measured, the ionization time is adjusted to an appropriate value, and a full scan is recorded. This function is called automatic gain control, or AGC $[10,11]$, and occurs on a continuous basis. High concentrations of materials eluting into the trap result in low ionization times being selected and vice versa. The ionization time for the full scan is stored and the total ion current trace is corrected postrun. The purpose of AGC is to avoid the build-up

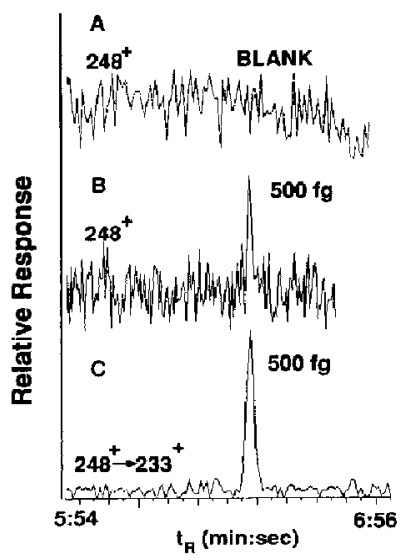

Figure 2. Capillary GC/MS and GC/MS/MS of standard TE: (a) Blank solvent injection in the mass selective storage mode $(m / z 248)$, (b) $500 \mathrm{fg}$ TE analyzed in the same fashion, (c) $500 \mathrm{fg}$ TE by capillary GC/MS/MS monitoring $248^{+} \rightarrow 233^{+}$. of space charge in the ion trap. It is important to note that this procedure (AGC) is also critical to the quantitation of analytes with unknown concentrations.

In this work some type of AGC function to optimize the population of parent ions isolated after the rf- and dc-voltage sequence would be ideal. However, no such algorithms exist for the $\mathrm{rf} / \mathrm{dc}$ method. Rf/dc is required [6] for analysis of crude extracts. Because we did not have access to a continuously variable ionization pulse for any $\mathrm{rf} / \mathrm{dc}$ or MS/MS applications, parent ion populations had to be optimized a different way.

Only fixed ionization times could be selected for each of these experiments. In constructing a calibration line over two orders of magnitude, however, this fixed ionization time was set to various values, based on the known analyte concentrations in the spiked calibration standards (Table 1). We also made use of a FORTH program to interactively change this value to an ionization time appropriate for the internal standard concentration after the analyte eluted $[6,12]$.

This is demonstrated in Figure 3, where the comparison of two chromatograms obtained for splitless injection of $250 \mathrm{pg}$ of TE and $2.5 \mathrm{ng} \mathrm{TE}$ is shown. Each sample also contained $5.4 \mathrm{ng}$ of internal standard. The peak areas for the analyte are similar in Figure $3 a$ and b. Notice that analyte and internal standard areas in Figure 3a are also similar. This is the effect of scaling the ionization time to the expected concentrations.

\section{Calibration Using Pure Standards}

Some of our initial results on quantitative capillary GC ion trap mass spectrometry methodologies [12] were derived from the use of split injections of these same compounds. Only $1 \%$ of a sample injection entered the capillary column but the standard solutions were 100 times more concentrated than used in this study. Therefore, the basic quantitative performance of the ion trap mass spectrometer was established for the $\mathrm{rf} / \mathrm{dc}-\mathrm{MS} / \mathrm{MS}$ approach, without the complications of high matrix or high solvent loads associated with trace analyses. Relative standard deviations (RSDs), of about $4 \%$, had been observed at both the low and high ends of the calibration line. A high correlation coefficient, $r=.9998$, was obtained [12].

By using solutions of pure standards in the current example, but 100 times more dilute than in our previous work, splitless injection of $2-\mu \mathrm{L}$ aliquots contain-

Table 1. Ionization pulse length versus amount injected for $\mathrm{TE}^{\mathrm{a}}$

\begin{tabular}{llllllll}
\hline $\begin{array}{l}\text { Amount TE } \\
\text { on-column (ng) }\end{array}$ & .100 & .251 & .502 & 1.00 & 2.51 & 5.02 & 10.0 \\
$\begin{array}{l}\text { lonization } \\
\text { time }(\mu \mathrm{s})\end{array}$ & 10,000 & 4000 & 2000 & 1000 & 400 & 200 & 100 \\
\hline
\end{tabular}

${ }^{a}$ All samples had 5.4-ng internal standard ienized for $200 \mu \mathrm{s}$. 


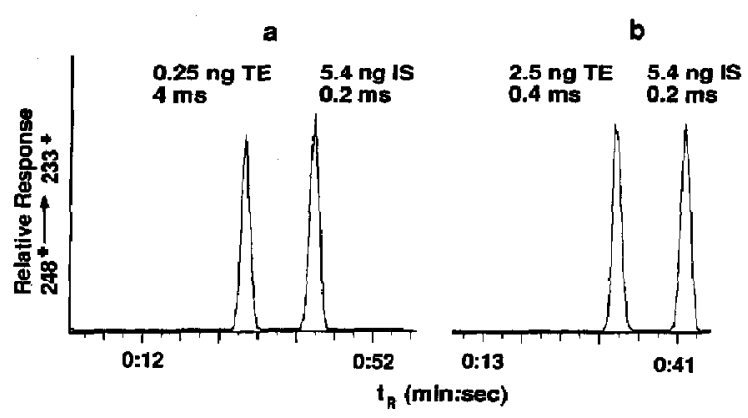

Figure 3. Capillary GC/MS/MS analysis of pure calibration standards in the full product ion scan mode. The amount injected splitless and the ionization time used are indicated over the peaks. The selected reaction $248^{+} \rightarrow 233^{+}$is reconstructed postrun. Retention times are relative to the time of activating the Forth program, not the injection time. (IS-internal standard).

ing $100 \mathrm{pg}-10 \mathrm{ng}$ of TE and $5.4 \mathrm{ng}$ internal standard were made. Full-scan product ion spectra were collected. In practice there is no need to do selected reaction monitoring on the trap because the family of product ions is trapped and scanning is rapid (18 $\mathrm{ms} / 100 \mathrm{u}$ is typical). Reconstructed reaction chromatograms $\left(248^{+} \rightarrow 233^{+}\right)$were plotted postrun. The ratio of the chromatographic peak areas was scaled inversely to the ionization time used and plotted versus the mass ratio of analy te to internal standard. The calibration line has a distinct curvature (Figure 4).

Because this calibration had previously displayed high linearity on other types of instruments $[12,13]$, we became suspicious of the accuracy of the selected ionization times. These times are used as scaling factors in constructing the calibration lines. Careful measurement with an oscilloscope showed that ion-

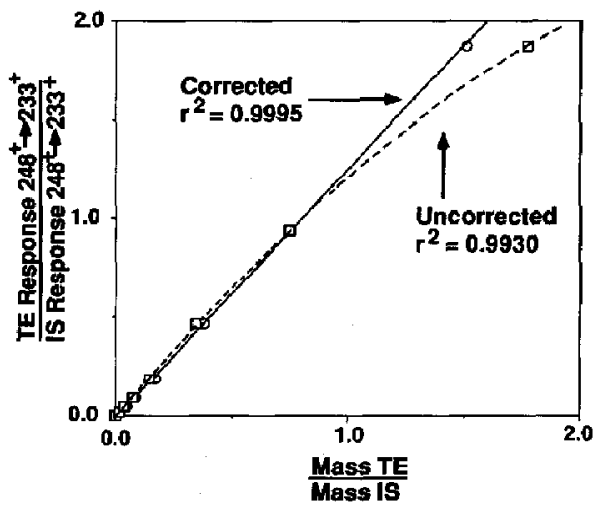

Figure 4. Calibration lines derived by plotting chromatographic peak area ratios of TE to IS versus their respective mass ratios in the capillary GC/MS/MS mode. The raw ratios, obtained from data as in Figure 2, are multiplied by the factor (ionization time intemal standard)/(ionization time TE). The corrected line is derived from oscillographically measured ionization times. ization times specified in the software tables did not match those produced by the instrument hardware.

A new calibration line was constructed by using scaling factors based on these accurately measured ionization times and was found to have improved linearity $\left(\mathrm{r}^{2}=.9995\right.$, Figure 4$)$. This correction procedure had not been necessary in our previous work [12]. If distinct curvature is observed in a regression line on the ion trap mass spectrometer, it is recommended that ionization times are checked, as described above. Over the past year, the relationship between computer-selected $(x)$ and observed ionization time $(y)$ has been stable and is defined by the relationship $y=1.0652 x+49.28$ over the range $25-10,000 \mu \mathrm{S}\left(r^{2}=1.000\right)$ on our instrument.

\section{Calibration from Plasma}

Analysis of a plasma extract is shown in Figure 5 for a sample spiked at $1 \mathrm{ppb}$ of TE. The upper trace shows the total product ion current versus time. It is suggested that this is qualitatively similar to the appearance of a selected ion monitoring (or in the case of the ion trap, selective mass storage) trace of $\mathrm{m} / z 248$ because the efficiency of converting parent ions of $m / z 248$ to product ions approaches $100 \%$. There are many chromatographic peaks present in the total ion trace and the analyte appears on a rising baseline. After elution of the analyte the fixed ionization time was changed via the FORTH program and a sharp drop in the ion current occurs $\left(t_{R}=6.5 \mathrm{~min}\right)$. The internal standard, at $50 \mathrm{ppb}$, is interference-free, based on blank analyses.

In the lower trace the ion current for the specific reconstructed reaction of $248^{+} \rightarrow 233^{+}$is shown. The

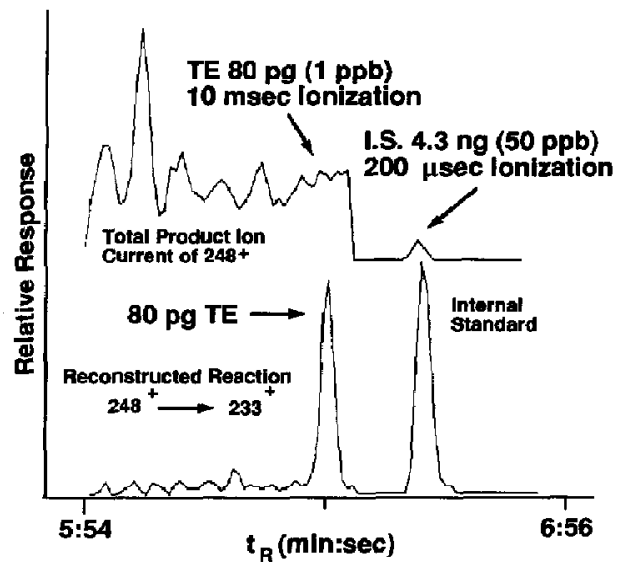

Figure 5. Capillary GC full-scan MS/MS analysis of an extract of plasma initially spiked at $1 \mathrm{ng} / \mathrm{g}$. The ionization time for TE is $10 \mathrm{~ms}$. The upper trace is a sum of all product ion current produced as a function of time. The lower trace is reconstructed for product ions of $m / z 233$ only. 
trace is very "clean," showing only the peaks for the analyte and the internal standard. Blank plasma extracts showed no responses above the baseline noise for TE or the internal standard for this reaction. The relative response near the analyte peak in this trace is about $10 \%$ of the upper trace; showing that significant ion current is carried by other components of the same nominal mass of $248 \mathrm{u}$.

Previously we demonstrated that $\mathrm{rf} / \mathrm{dc}$ was necessary prior to MS/MS of crude extracts of tissue homogenates [6]. By using $\mathrm{rf}$ voltages only, all ions above a certain mass are stored prior to MS/MS, resulting in less rejection of spurious matrix ions in comparison to the $\mathrm{rf} / \mathrm{dc}$ method. The MS/MS experiment is adversely affected as well, giving poor product ion production. The analyte peak is barely discernible in the chromatogram. We hypothesize that this is due to the level of space charge present. We found the same to be true in the analysis of these plasma extracts, with a failed MS/MS result in the MS/MS approach using rf-voltages only (Figure 6).

The calibration line derived from extraction of these plasma samples had a linear correlation, $r^{2}$, of 1.000 and a regression line $y=.804 x+.002$, in good agreement with previous results. The RSDs were similar. We then moved to the analysis of a few clinical samples with unknown concentrations of drug and a question immediately arose: What ionization time does one select for the analyte in such samples?

Because AGC is not available for these experiments, the answer is to run the sample twice, which is a clear drawback. After a test run is completed, one can then select an appropriate ionization time based on the estimated concentration of the analyte. In fact, this is what AGC accomplishes in real-time GC/MS, using the total ion current of a short test scan to set the ionization time. An initial evaluation of clinical samples (submitted blind to us) was carried out.

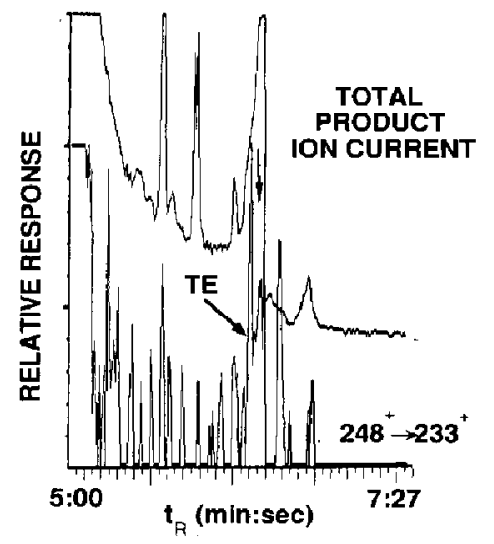

Figure 6. Attempted analysis of the sample shown in Figure 5, but using only if voltages. All ions above $m / z 245$ are stored prior to attempted dissociation of $m / z 248$. Space charge effects may account for the failed analysis.
Agreement of TE concentrations within $15 \%$ was found between the TSQ and ion trap mass spectrometer results.

A more thorough comparison of the two methods will follow in a subsequent article. Briefly though, in our opinion, full-scan, product ion spectra, available from the ion trap, but not the TSQ at these levels, may not provide any particular advantage if one is interested only in quantitation of a target analyte.

On the other hand, we recently described an interesting discovery of putative prostaglandin metabolites that would not have been made without the full-scan MS/MS sensitivity of the ion trap [17]. In that case, five different reaction chromatograms were reconstructed postrun from a single capillary GC/full-scan MS/MS run on a sample estimated to contain about 1 ng of $\mathrm{PGE}_{2}$. In each of the five reaction chromatograms peaks were observed at the same retention time at several points during the chromatographic run. This suggested the presence of several compounds of unique structure in the sample, yet all possessing a common substructure found in the parent ion chosen for MS/MS.

We note two capillary GC/MS applications in which the ion trap detector was used with selective chemical ionization (CI). Analysis of drugs, down to the low parts-per-billion level, in crude extracts of plasma or urine, was demonstrated $[15,16]$. The success of those methods is partly due to the cleaner nature of basic extracts of biological samples but also to the selectivity of the acetonitrite $\mathrm{Cl}$ of the basic drugs. Quantitation was achieved via automatic reaction control $[15,16]$, a $\mathrm{Cl}$ analogy of $\mathrm{AGC}$, and by using an internal standard separated by retention time. Finally, there are on-going attempts to improve our initial results with coeluting stable isotope-labeled internal standards [18].

\section{Conclusions}

As a high sensitivity, quantitative MS/MS device for analysis of crude extracts, the ion trap mass spectrometer is still evolving. For narrow capillary GC peaks, internal standards separated by retention time are the only practical choice. The lack of an AGC function for the more complex rf/dc-MS/MS scan functions, necessary for crude extract analysis, is a hindrance to practical quantitative analyses. With the development of a version of $A G C$ for these applications, the ion trap may compete with triple quadrupole tandem mass spectrometers as a more sensitive quantitative MS/MS device. This remains to be seen, however, and will likely be compound-dependent, in our opinion. It is well established at this point that the ion trap is a much more sensitive, full-product ion scan MS/MS device. Taken together with the $\mathrm{MS}^{n}$ capabilities of the trap, these considerations make it an attractive option for future qualitative applications like peptide sequencing. 


\section{References}

1. Johnson, J. V.; Yost, R. A.; Kelley, P. E.; Bradford, Donald C. Anal. Chem. 1990, 62, 2162-2172.

2. March, R. E.; Hughes, R. J. Quadrupole Storage Mass Spectrometry: Wiley: New York, 1989.

3. Strife, R. J.; Kelley, P. E.; Weber-Grabau, M. Rapid Commun. Mass Spectrom. 1988, 2, 105-109.

4. Pannell, L. K.; Pu, Q.-L.; Fales, H. M.; Mason, R. T. Attal Chem. 1989, 61, 2500-2503.

5. McLuckey, S. A.; Glish, G. L.; Asano, K. G.; Van Berkel, G. J. Anal. Chem. 1988, 60, 2312-2314.

6. Strife, R. J.; Simms, J. R. Anal. Chem. 1989, 61, 2316-2319.

7. Fulford, J. E.; March, R. E. Int. J. Mass Spectrom. Ion Phys. 1978, 26, 155-162.

8. Bonner, R. F, Int, I. Mass Spectrom. Ion Phys. 1977, 23, 249-257.

9. Louris, J. N.; Brodbelt-Lustig, J.; Cooks, R. G.; Glish, G. L.; Van Berkel, G. J.; McLuckey, S. A. Int. J. Mass Spectrom. Ion Phys. 1990, 96, 117-137.
10. Stafford, G. C.; Taylor, D. M.; Bradshaw, S. C.; Syka, J. E. P.; Ulrich, M. Presented at the 35th ASMS Conference on Mass Spectrometry and Allied Topics; Denver, CO, May 24-29, 1987.

11. Yost, R. A.; McClennen, W.; Meuzelaar, H. L. C. Application Report \# 209; Finnigan-MAT, San Jose, CA, 1988.

12. Strife, R. J.; Simms, J. R.; Lacey, M. P. J. Am. Mass Spectrom. 1990, 1, 265-271.

13. Dobson, R. L. M.; Neal, D. M.; DeMark, B. R.; Ward, S. R. Anal Chem. 1990, 62, 1819-1824.

14. Technical Report \#608; Fìnnigan-Mat, San Jose, CA, 1988.

15. Lim, H. K.; Sakashita, C. O.; Foltz, R. L. Rapid Commun. Mass Spectrom. 1988, 2, 129-131.

16. Shetly, H. U.; Daly, E. M.; Soncrant, T. T.; Greig, N. H.; Rapoport, S. I. J. Am. Soc. Mass Spectrom. 1991, 2, 168-173.

17. Strife, R. J.; Simms, J. R.; Stepp, E. H. Proceedings of the 38th ASMS Conference on Mass Spectrometry and Allied Topics: Tucson, AZ, June 3-8, 1990.

18. Kleintop, B. L.; Yost, R. A.; Abolin, Craig R. Praceedings of the 39th ASMS Conference on Mass Spectrometry and Allied Topics; Nashville, TN, May 19-24, 1991. 